Pacific Journal of Mathematics

ON ELEMENTARY IDEALS OF PROJECTIVE PLANES IN THE
4-SPHERE AND ORIENTED @-CURVES IN THE 3-SPHERE 


\title{
ON ELEMENTARY IDEALS OF PROJECTIVE PLANES IN THE 4-SPHERE AND ORIENTED $\theta$-CURVES IN THE 3-SPHERE
}

\author{
SHIN'ICHI KINOSHITA
}

The concept of an infinite cyclic covering has been applied to knot theory. In this paper that of a finite cyclic covering is considered. This enable us to study such cases as projective planes in the 4 -sphere and oriented $\theta$-curves in the 3-sphere. Some properties of elementary ideals of these cases are examined. The technique of free differential calculus is used, instead of that of coverings.

Let $L$ be a polyhedron in an $n$-sphere $S^{n}(n>1)$ that does not separate $S^{n}$, and let $G_{L}$ be the fundamental group of $S^{n}-L$. We use the additive group $J_{p}$ of integers modulo $p$ as the coefficient group for homology. Let $l$ be an $(n-2)$-dimensional cycle on $L$. Let $H_{p}$ be the multiplicative cyclic group of order $p$, generated by $t$. Then, there is a homomorphism $\psi$ of $G_{L}$ into $H_{p}$ such that for each $g \in G_{L}$,

$$
g^{\gamma / \gamma}=t^{\operatorname{link}(g, l)},
$$

where link $(g, l) \in J_{p}$ is the linking number between $g$ and $l$ in $S^{n}$.

Using Fox's free differential calculus ([1], [2]), we associate to $\psi$ a sequence of elementary ideals $E_{d}\left(G_{L}, \psi\right)$ of the group $G_{L}$, evaluated in the group ring $J H_{p}$ of $H_{p}$ over integers $J$. This sequence of elementary ideals depends only on $G_{L}$ and $\psi$, and hence it depends only on the position of $l$ on $L$ in $S^{n}$. We shall denote it by $E_{d}(l)$. If $l$ and $l^{\prime}$ are homologous on $L$, then $E_{d}(l)=E_{d}\left(l^{\prime}\right)$ for every $d$.

In this paper we apply these elementary ideals $E_{d}(l)$ to the study of the position of $L$ in $S^{n}$. The following two cases of $E_{d}(l)$ are considered: (1) $L$ is a projective plane in $S^{4}$ and $p=2$, and (2) $L$ is a $\theta$-curve in $S^{3}$ and $p=3$.

1. Miscellanea. Let $\sigma(t)=1+t+\cdots+t^{p-1} \in J H_{p}$.

THEOREM 1. If $\psi$ is onto, then $E_{0}(l) \subset(\sigma(t))$ in $J H_{p}$.

Proof. It is proved in [2] that

$$
E_{0}\left(H_{p}, i d\right)=(\sigma(t)),
$$

where $i d$ is the identity isomorphism of $H_{p}$. From the diagram

$$
G_{L} \stackrel{\psi}{\longrightarrow} H_{p} \stackrel{i d}{\longrightarrow} H_{p}
$$


where $\psi$ is onto, and Theorem 1 in [4], it follows that $E_{0}(l) \subset(\sigma(t))$ in $J H_{p}$.

Now assume that $\psi$ is onto, and let $E_{0}(l)=\sigma(t) E(l)$. Let $\omega=e^{2 \pi i / p}$ and let $J[\omega]$ be the ring of all complex numbers of the form $\sum_{i=0}^{p-1} a_{i} \omega^{i}$, where $a_{i} \in J(i=0,1, \cdots, p-1)$. A homomorphism * of $H_{p}$ into $J[\omega]$ is defined by $t^{*}=\omega$. We naturally extend $*$ to a ring homomorphism of $J H_{p}$ onto $J[\omega]$. Though $E_{0}(l)^{*}=(0)$, sometimes $E(l)^{*}$ is a nontrivial ideal in $J[\omega]$.

A trivializer of a group $G$ is a homomorphism of $G$ onto the trivial group that consists of only one element. Any trivializer will be denoted by the same notation $\circ$ in this paper. Further the group ring $J G^{\circ}$ will be identified with $J$.

2. Projective planes in $\mathrm{S}^{4}$. Let $P$ be a polyhedral projective plane in $S^{4}$. By the Alexander duality theorem, the abelianization of the fundamental group $G_{P}$ of $S^{4}-P$ is a cyclic group of order 2 . We use $J_{2}$ as the coefficient group for homology. Let $l$ be a 2-cycle on $P$.

$$
\text { Theorem 2. }\left\{\begin{array}{l}
E_{0}(l)^{\circ}=(2) \text { and } \\
E_{d}(l)^{\circ}=(1) \text {, if } d>0, \text { in } J .
\end{array}\right.
$$

Proof. This follows to Theorem 2 in [4].

A projective plane $P$ has only two cycles. First let $l_{0}$ be the trivial one.

$$
\text { Theorem 3. }\left\{\begin{array}{l}
E_{0}\left(l_{0}\right)=(2) \text { and } \\
E_{d}\left(l_{0}\right)=(1) \text {, if } d>0, \text { in } \mathrm{JH}_{2} .
\end{array}\right.
$$

Proof. The proof is similar to that of Theorem 3 in [4].

Now let $l$ be the nontrivial 2-cycle on $P$, i.e., the fundamental cycle for $J_{2}$-orientation of $P$. Since the homomorphism $\psi$ is onto in this case, by Theorem 1 we have $E_{0}(l) \subset(1+t)$ in $J H_{2}$. Let $E_{0}(l)=$ $(1+t) E(l)$.

THEOREM 4. $E(l)^{\circ}=(1)$ in $J$.

Proof. Since

$$
(2)=E_{0}(l)^{\circ}=(1+t)^{\circ} E(l)^{\circ}=(2) E(l)^{\circ}
$$

in $J$, we have $E(l)^{\circ}=(1)$ in $J$.

Further $E(l)^{*} \subset J$ is also an invariant of $P$ in $S^{4}$.

TheOREM 5. The ideal $E(l)^{*}$ in $J$ is generated by an odd integer. 
Proof. Let $E(l)$ be generated by $a_{\imath}+b_{i} t(i=1,2, \cdots, n)$ in $J H_{2}$. Assume on the contrary that $E(l)^{*}$ is not generated by an odd integer. Then we have $a_{i}-b_{i}=0 \bmod .2$ for every $i$. From this it follows that $a_{i}+b_{\imath}=0 \bmod .2$ for every $i$. Hence we have $E(l)^{\circ} \neq(1)$ in $J$ which contradicts Theorem 4.

EXAMPLE 1. Let $f(t)$ be an integral polynomial with $f(1)=1$. Then, for each $f(t)$ there is a polyhedral, locally flat projective plane $P_{f}$ in $S^{4}$, where the odd natural number $|f(-1)|$ is a topological invariant of $P_{f}$ in $S^{4}$ (see [3]). In these example, it is easy to see that for the nontrivial 2-cycle $l$ on $P_{f}$ we have $E(l)=(f(t))$ in $J H_{2}$, where $f(t)$ is considered as an element of $J H_{2}$. Further we have $E(l)^{*}=(f(-1))$ in $J$.

3. $\theta$-curves in $\mathrm{S}^{3}$. Let $P$ and $Q$ be two distinct points in $S^{3}$ and let $a_{1}, a_{2}$, and $a_{3}$ be three polygonal ares from $P$ to $Q$, which are mutually disjoint to each other except at $P$ and $Q$. Then $L=a_{1} U$ $a_{2} \cup a_{3}$ is called a $\theta$-curve in $S^{3}$. Further, if each of these three arcs is oriented from $P$ to $Q$, then $L$ is called an oriented $\theta$-curve in $S^{3}$. From now on we use $J_{3}$ as the coefficient group for homology.

Let $L$ be a $\theta$-curve in $S^{3}$. Then the abelianization of the fundamental group of $S^{3}-L$ is a free abelian group of rank 2 . Let $l$ be a 1-cycle on $L$.

Theorem 6. $\left\{\begin{array}{l}E_{0}(l)^{\circ}=E_{1}(l)^{\circ}=(0) \text { and } \\ E_{d}(l)^{\circ}=(1) \text {, if } d>1 \text {, in } J .\end{array}\right.$

Proof. This follows to Theorem 9 in [4].

THEOREM 7. $E_{0}(l)=E_{1}(l)=(0)$ in $J H_{3}$.

Proof. This follows to corollary of Theorem 7 in [4].

Now let $L$ be an oriented $\theta$-curve in $S^{3}$. Then there is a nontrivial 1-cycle $l$ on $L$ such that the coefficient of $l$ for each oriented 1 -simplex of $L$ is $1 \in J_{3}$. The 1 -cycle $l$ is called the fundamental cycle for the $J_{3}$-orientation of $L$. Then, $E_{2}(l)$ in $J H_{3}$ and $E_{2}(l)^{*}$ in $J[\omega]$, where $\omega=e^{2 \pi i / 3}$, are topological invariants of the oriented $\theta$-curve $L$ in $S^{3}$.

ExAmple 2. Let $L$ be the example of an oriented $\theta$-curve in [4], where the orientation of $L$ is given as shown in the figure in [4]. Let $l$ be the fundamental cycle for this $J_{3}$-orientation of $L$. Then we have 


$$
\left\{\begin{array}{l}
E_{0}(l)=E_{1}(l)=(0), \\
E_{2}(l)=\left(t^{2}+t+1,2\right) \text { and } \\
E_{d}(l)=(1), \text { if } d>2,
\end{array}\right.
$$

in $J H_{3}$ and $E_{2}(l)^{*}=(2)$ in $J[\omega]$.

THEOREM 8. Let $f(t) \in J H_{3}$ with $f(1)=1$. Then there is an oriented $\theta$-curve $L$ in $S^{3}$ such that for the fundamental cycle $l$ for the $J_{3}$-orientation of $L$ we have

$$
\left\{\begin{array}{l}
E_{0}(l)=E_{1}(l)=(1), \\
E_{2}(l)=(f(t)) \text { and } \\
E_{d}(l)=(1), \text { if } d>2, \text { in } J H_{3} .
\end{array}\right.
$$

Proof. Let $f(\tau) \in J H$ with $f(1)=1$, where $H$ is an infinite cyclic multiplicative group generated by $\tau$. Then there is an example of a $\theta$-curve $L_{1}$ and a 1 -cycle $l_{1}$ on $L_{1}$ such that

$$
\left\{\begin{array}{l}
E_{0}\left(l_{1}\right)=E_{1}\left(l_{1}\right)=(1), \\
E_{2}\left(l_{1}\right)=(f(\tau)) \text { and } \\
E_{d}\left(l_{1}\right)=(1), \text { if } d>2,
\end{array}\right.
$$

in $J H$ (see [5]). The coefficients of $l_{1}$ on $L_{1}$ are distributed as shown in Fig. 1. Note that arcs in the outside of the cube shown by dotted lines in the figure are possibly complicated. Now the sequence of elementary ideals remains invariant, even if $L_{1}$ is "blown up" to a cube with 2 handles. Then the 1-cycle $l_{2}$ on $L_{2}$ as shown in Fig. 4 has the same sequence of elementary ideals to that of $l_{1}$ on $L_{1}$. Considering $l_{2}$ in the homology of integers modulo 3 , we have an example of a 1-cycle $l$ on $L$ as shown in Fig. 5. The 1 -cycle $l$ is the fundamental cycle of a $J_{3}$-orientation of the $\theta$-curve $L$ and for each $d$ we have $E_{d}(l)=E_{d}\left(l_{1}\right)^{\prime}$ in $J H_{3}$, where ' is a ring homomorphism

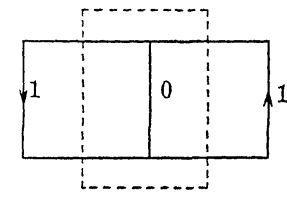

Fig. 1

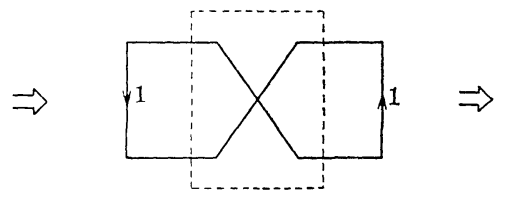

Fig. 2

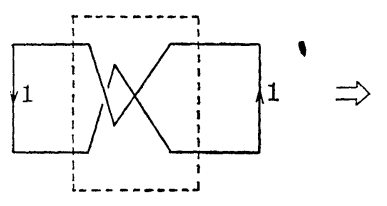

Fig. 3

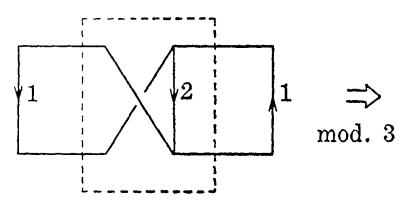

Fig. 4

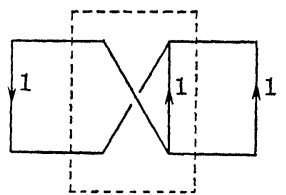

Fig. 5 
of $J H$ onto $\mathrm{JH}_{3}$ defined by $\tau^{\prime}=t$. Now the theorem can be seen easily.

\section{REFERENCES}

1. R. H. Fox, Free differential calculus, I, Ann. of Math., 57 (1953), 547-560.

2. —- Free differential calculus, II, Ann. of Math., 59 (1954), 196-210.

3. S. Kinoshita, On the Alexander polynomials of 2-spheres in a 4-sphere, Ann. of Math., 74 (1961), 518-531.

4. ㄴ On elementary ideals of polyhedra in the 3-sphere, Pacific J. Math., 42 (1972), 89-98.

5. - On elementary ideals of $\theta$-curves in the 3-sphere and 2-links in the 4sphere, Pacific J. Math., 49 (1973), 127-134.

Received July 16, 1974.

Florida State University 



\title{
PACIFIC JOURNAL OF MATHEMATICS
}

\author{
EDITORS
}

RICHARD ARENS (Managing Editor)

University of California

Los Angeles, California 90024

R. A. Beaumont

University of Washington

Seattle, Washington 98105
J. DugundJI

Department of Mathematics University of Southern California Los Angeles, California 90007

D. Gilbarg and J. Milgram

Stanford University

Stanford, California 94305

\section{ASSOCIATE EDITORS}

E. F. BECKENBACH

B. H. NeUManN

F. WOLF

K. YosHIDA

\section{SUPPORTING INSTITUTIONS}

UNIVERSITY OF BRITISH COLUMBIA

GALIFORNIA INSTITUTE OF TECHNOLOGY

UNIVERSITY OF CALIFORNIA

MONTANA STATE UNIVERSITY

UNIVERSITY OF NEVADA

NEW MEXICO STATE UNIVERSITY

OREGON STATE UNIVERSITY

UNIVERSITY OF OREGON

OSAKA UNIVERSITY
UNIVERSITY OF SOUTHERN CALIFORNIA

STANFORD UNIVERSITY

UNIVERSITY OF TOKYO

UNIVERSITY OF UTAH

WASHINGTON STATE UNIVERSITY

UNIVERSITY OF WASHINGTON

AMERICAN MATHEMATICAL SOCIETY NAVAL WEAPONS CENTER 


\section{Pacific Journal of Mathematics}

\section{Vol. 57, No. $1 \quad$ January, 1975}

Keith Roy Allen, Dendritic compactification ......................... 1

Daniel D. Anderson, The Krull intersection theorem ..................... 11

George Phillip Barker and David Hilding Carlson, Cones of diagonally dominant

matrices .............................................

David Wilmot Barnette, Generalized combinatorial cells and facet splitting ........

Stefan Bergman, Bounds for distortion in pseudoconformal mappings ............

Nguyên Phuong Các, On bounded solutions of a strongly nonlinear elliptic

equation ...............................................

Philip Throop Church and James Timourian, Maps with 0-dimensional critical

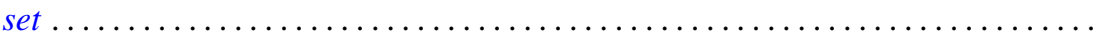

G. Coquet and J. C. Dupin, Sur les convexes ubiquitaires ................. 67

Kandiah Dayanithy, On perturbation of differential operators............... 85

Thomas P. Dence, A Lebesgue decomposition for vector valued additive set

functions .............................................. 91

John Riley Durbin, On locally compact wreath products.................. 99

Allan L. Edelson, The converse to a theorem of Conner and Floyd ............. 109

William Alan Feldman and James Franklin Porter, Compact convergence and the

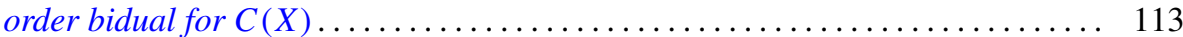

Ralph S. Freese, Ideal lattices of lattices......................... 125

R. Gow, Groups whose irreducible character degrees are ordered by divisibility ... 135

David G. Green, The lattice of congruences on an inverse semigroup ............ 141

John William Green, Completion and semicompletion of Moore spaces .......... 153

David James Hallenbeck, Convex hulls and extreme points of families of starlike and close-to-convex mappings .................................. 167

Israel (Yitzchak) Nathan Herstein, On a theorem of Brauer-Cartan-Hua type ...... 177

Virgil Dwight House, Jr., Countable products of generalized countably compact spaces ............................................ 183

John Sollion Hsia, Spinor norms of local integral rotations. I . . . . . . ......... 199

Hugo Junghenn, Almost periodic compactifications of transformation

semigroups....................................

Shin'ichi Kinoshita, On elementary ideals of projective planes in the 4-sphere and oriented $\Theta$-curves in the 3 -sphere ............................ 217

Ronald Fred Levy, Showering spaces............................ 223

Geoffrey Mason, Two theorems on groups of characteristic 2-type............. 233

Cyril Nasim, An inversion formula for Hankel transform ................... 255

W. P. Novinger, Real parts of uniform algebras on the circle ................ 259

T. Parthasarathy and T. E. S. Raghavan, Equilibria of continuous two-person

games............................................... 265

John Pfaltzgraff and Ted Joe Suffridge, Close-to-starlike holomorphic functions of several variables ....................................... 271

Esther Portnoy, Developable surfaces in hyperbolic space .................. 281

Maxwell Alexander Rosenlicht, Differential extension fields of exponential type . . . 289

Keith William Schrader and James Lewis Thornburg, Sufficient conditions for the

existence of convergent subsequences ........................... 301

Joseph M. Weinstein, Reconstructing colored graphs .................. 307 